\title{
INFLUENCE OF FOLIAR NUTRITION ON YIELD AND YIELD COMPONENTS OF DURUM WHEAT (TRITICUM DURUM DESF.) GROWN IN SYSTEM OF ORGANIC PRODUCTION
}

\author{
ZEČEVIĆ, V. ${ }^{*}{ }^{*}$ - MiLENKOVIĆ, S. ${ }^{2}$ - BoŠKOVIĆ, J. ${ }^{3}$ - RolJEVIĆ NiKOLIĆ, S. ${ }^{4}$ - LUKOVIĆ, K. ${ }^{5}$ - \\ ĐORĐEVIĆ, R. ${ }^{1}-$ KNEŽEVIĆ, D. ${ }^{6}$ \\ ${ }^{1}$ Institute for Vegetable Crops, Karadjordjeva 71, 11420 Smederevska Palanka, Serbia \\ ${ }^{2}$ Faculty of Ecological Agriculture, Educons University, Vojvode Putnika 85-87, \\ 21208 Sremska Kamenica, Serbia
}

${ }^{3}$ Faculty of Economy and Engineering Management, University Business Academy, Cvećarska 2, Novi Sad, Serbia

${ }^{4}$ Institute of Agricultural Economics, Volgina 15, 11050 Belgrade, Serbia

${ }^{5}$ Centre for Small Grains, Save Kovačevića 31, 34000 Kragujevac, Serbia

${ }^{6}$ Faculty of Agriculture, University of Pristina, Kosovska Mitrovica-Lešak, Kopaonička bb Lešak, Kosovo and Metohia, Serbia

*Corresponding author

e-mail:vzecevic@institut-palanka.rs

(Received $1^{\text {st }}$ Jul 2021; accepted $28^{\text {th }}$ Oct 2021)

\begin{abstract}
This paper examines the effect of foliar nitrogen nutrition on seven durum wheat genotypes produced according to the principles of organic production. The experiments were performed during two growing seasons on organic farm in Čačak, Serbia. Three different N fertilization strategies have been compared: T1- control without $\mathrm{N}$ application after the stem elongation stage; $\mathrm{T} 2$ - foliar $\mathrm{N}$ fertilization at the beginning of heading, one spray with $0.3 \%$ organic fertilizer Trainer (5\% $\mathrm{N}$ and $31 \%$ amino acids); T3- foliar $\mathrm{N}$ fertilization in both stages, heading and anthesis with $0.3 \%$ organic fertilizer Trainer $(5 \% \mathrm{~N})$ each. Analysis of grain yield and yield components (number of spikes $\mathrm{m}^{-2}$, number of grains per spike and thousand grain weight (TGW) was performed. The number of spikes per square meter, number of grains spike $^{-1}$, TGW, and grain yield significantly increased $(\mathrm{P} \leq 0.01)$ with different levels of fertilizers. Foliar fertilization had a significant effect on yield in both growing seasons. Grain yield, on average for all genotypes and years after spraying were $22 \%$ (one $\mathrm{N}$ treatment), and $54 \%$ (two $\mathrm{N}$ treatments) higher than in the control. Grain yield was about $26 \%$ higher in two $\mathrm{N}$ treatments than in one $\mathrm{N}$ treatment. Grain yield was positively correlated with other traits (TGW, number of spikes $\mathrm{m}^{-2}$ and number of grains/spike).
\end{abstract}

Keywords: durum wheat, organic management, $N$ treatment, grain yield, correlations

Abbreviations: ANOVA, analysis of variance; G, genotype; LSD, least significant difference; T, N treatment; T1, control without $\mathrm{N}$ application after the stem elongation stage; T2, foliar liquid $\mathrm{N}$ fertilization at the beginning of heading; T3, foliar liquid $\mathrm{N}$ fertilization in both stages, heading and anthesis; TGW, thousand grain weight; Y, year.

\section{Introduction}

Durum wheat (Triticum durum Desf.) is a plant species represented on only 8 to $10 \%$ of all wheat growing areas. Despite of its small area, durum wheat is economically important species because of its unique characteristics and end products. Durum wheat grain is characterized by high protein and gluten content which makes it suitable for the 
production of a variety of food products, such as pasta, couscous, bread, etc. (Rao et al., 2010). Compared to the ordinary wheat, durum has a larger grain of higher absolute mass, a much higher content of yellow pigment, and relatively higher protein inelastic gluten contents which reduces the elasticity of the dough ( $\mathrm{Li}$ et al., 2013). Pasta is the most common end product of durum wheat consumed in Europe, North America and the former USSR (Mohammadi et al., 2011). Durum wheat is one of the most widespread plant species in arid conditions and Mediterranean environments, where high temperatures limit the productivity of genotypes (Araus et al., 2002), although this condition provides the ability to produce high quality durum.

Creating durum wheat genotypes with increased productivity in arid conditions has been an important aspect of many breeding programs. Durum wheat corresponds to the warmer regions, where annual rainfall of $350-450 \mathrm{~mm}$ is concentrated in the vegetative phase, high temperatures after fertilization, less humidity (improves grain quality), occasional showers and prolonged sunny and warm weather in the grain filling phase.

In an organic production system, it is desirable that the varieties possess the following characteristics: efficiency of absorption and use of nutrients from the soil, good competitiveness with weeds, tolerance to climatic and environmental stresses, stability of yield and good quality of products (Lammerts van Bueren et al., 2002; Bošković et al., 2016; Branković et al., 2018). Organic durum wheat production is topical due to the increasing demands of consumers and the food industry for organic products.

Wheat grain yield is influenced by various factors, primarily the genotype characteristics, the soil fertility and the applied agro-technical measures. Studies have shown that nitrogen, in interaction with other elements of the mineral nutrition, is the main carrier of wheat grain yield and quality (Matković et al., 2015). The effect of nitrogen on wheat yield depends on the time of nitrogen application. The application of nitrogen with watering generally increases the yield beyond the degree of tillering, which is the number of spikes and grains per unit area (Langer, 1979). The application of nitrogen between stem elongation and flowering phase increases yield through increasing the percentage of productive tillering and number of grains in the spike. The application of nitrogen in the later stages of development increases yield by increasing grain mass and often by increasing the number of grains per spike (Kostić and Đokić, 1975). Tedone et al. (2014) found that $\mathrm{N}$ application efficiency and $\mathrm{N}$ recovery efficiency in wheat crops increased when $\mathrm{N}$ fertilizer is applied at the stem elongation phase, whereas high amounts of $\mathrm{N}$ at sowing time and tillering resulted in poor efficiency.

The application of balanced fertilizers is one of the most important factors for increasing crop yields. In wheat cultivation, the farmers are paying great attention only to $\mathrm{N}$ fertilisation but very often $\mathrm{P}$ and $\mathrm{K}$ application are partially or completely ignored (Arshadullah et al., 2015). Optimal doses of nitrogen for wheat yield change depending on climatic factors, soil fertility, crop rotation, fertilization in the previous period, etc. Favorable climatic performance in the filling phase of the kernels can favor both the accumulation of amidaceous and protein substances so the availability of nitrogen in the soil at this time is critical to the qualitative improvement of the grain (Branković et al., 2015a, 2016; Tedone et al., 2017).

The aim of this paper is investigation of influence of foliar nutrition on yield value and yield components variability in durum wheat grown in system of organic farming production. 


\section{Materials and methods}

\section{Plant material}

Seven genotypes were used in the experiment. Three varieties of winter durum wheat Windur (Germany), Žitka, KG Olimpik (Serbia), and four breeding lines KG-28-6, KG3405-03, KG-43-33-1, and KG-44-3-1 (Serbia) were grown during two growing seasons (2012/2013 and 2013/2014) at certified organic trial parcel which is located in Mršinci, in the Municipality of Čačak, Serbia $\left(20^{\circ} 30^{\prime} \mathrm{E}, 43^{\circ} 48^{\prime} \mathrm{N}, 220 \mathrm{~m}\right.$ a.s.l.). Origin of cultivar Windur is Germany, Žitka is the property of the Center for Agricultural and Technological Research, Zaječar (Serbia), and other investigated genotypes were selected in Small Grains Research Centre in Kragujevac (Serbia).

\section{Field trials and methods}

The field experiment was conducted in a randomized block design with three replications on plot of $5 \mathrm{~m}^{2}$ on the soil which belongs to the clay loam soil type. Experiment was carried out by the organic technology of scientific farming production of wheat. Potato was preceding crop in the first growing season (2012/2013), while bean was preceding in the second year (2013/2014). In autumn, starter fertilization was done with two tons of organic fertilizer Italpollina $(4: 4: 4)-80 \mathrm{~kg} \mathrm{ha}^{-1}$ of pure nitrogen $(\mathrm{N})$. The soil is cultivated only with rototiller. Sowing was done on November 6, 2012, and in October 25, 2013 with 600 seeds per square meter. The treatment of the crops during the growing seasons respected the principles of the organic farming.

At the tillering stage (in February 2013), crop was fertilized with $500 \mathrm{~kg} \mathrm{~h}^{-1}$ organic fertilizer Dix $10(10: 3: 3)$ - $50 \mathrm{~kg} \mathrm{ha}^{-1}$ of pure N. The fertilizers applied are produced at Hello Nature, former Italpollina (Italy). Three different strategies of $\mathrm{N}$ application in different phase of plant development have been compared: $\mathrm{T} 1$ - control without $\mathrm{N}$ application after the stem elongation stage; T2- foliar liquid $\mathrm{N}$ fertilization at the beginning of heading (Z 52 according to Zadox, 1974), 10 April 2013 and 14 April 2014, one spray with $0.3 \%$ organic fertilizer Trainer (5\% $\mathrm{N}$ and $31 \%$ amino acids); T3foliar liquid $\mathrm{N}$ fertilization in both stages heading ( $\mathrm{Z}$ 52) and anthesis- Z 60 (8 May 2013 and 12 May 2014), two sprays with $0.3 \%$ organic fertilizer Trainer according to the commercial recommended rate.

In full maturity stage of wheat, samples were taken from $\mathrm{m}^{-2}$ in three replications to determine the yield and yield components in both years. All spikes from $\mathrm{m}^{-2}$ were threshed on the Wintersteiger Hege 16 laboratory thresher in Laboratory of Small Grains Research Centre of Kragujevac. Grain yield, number of spikes per square meter, number of grains per spike and thousand grain weight (TGW) were determined. The number of grains per spike was determined from 30 random spikes, which were threshed manually.

\section{Soil analysis}

The topsoil samples were taken from the depth 0-30 cm. This depth was chosen as a zone of the most active root systems of observed crops. The samples were taken using a soil drill agrochemical probes. One composite sample represented 20-25 subsamples from random points in each sampling site. The soil samples were air-dried (room temperature), milled and sieved to a particle size of $<2 \mathrm{~mm}$, in accordance with ISO 11464: 2006. 


\section{Laboratory analysis}

All laboratory analyses were performed at the Laboratory for Soil and Agroecology of the Institute of Field and Vegetable Crops, Novi Sad, Serbia, accredited according to the standard ISO/IEC 17025: 2017 (Fig. 1). The $\mathrm{pH}$ value in 1:2.5 (v/v) suspension of soil in $1 \mathrm{M} \mathrm{KCl}$ and $\mathrm{H}_{2} \mathrm{O}$ was determined using a glass electrode, in accordance with the ISO method 10390:2005. The hydrolytic acidity values were determined according to Kappen (1929) in calcium acetate solution by titration method. The carbonate content as free $\mathrm{CaCO}_{3}$ was determined by the volumetric method ISO 10693:1995. The organic matter content (OM) was measured by the sulfochromic oxidation method ISO 14235:1998. The total $\mathrm{N}$ and $\mathrm{S}$ were determined by elemental analysis on the CHNS analyser VARIO El III according to AOAC 972.43 (2000) method. Readily available phosphorus $\left(\mathrm{P}_{2} \mathrm{O}_{5}\right)$ and available potassium $\left(\mathrm{K}_{2} \mathrm{O}\right)$ were extracted by ammonium lactate extraction (AL method), and measured by the means of spectrophotometry and flame photometry respectively (Egner and Riehm, 1955). Particle size distribution in the $<2 \mathrm{~mm}$ soil fraction was determined by the pipette method (Van Reeuwijk, 2002) and soil texture determined according to International Union of Soil Sciences (IUSS) system of classification of soil particles.

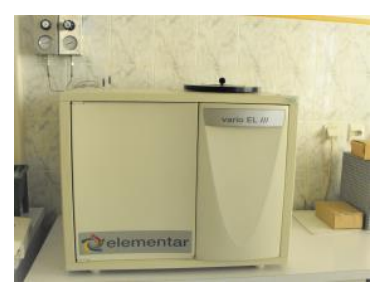

(a)

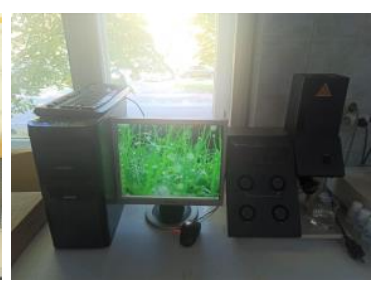

(b)

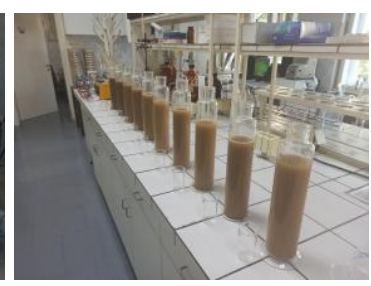

(c)

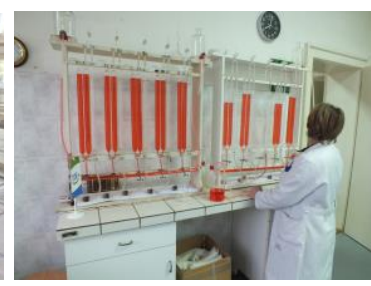

(d)

Figure 1. Equipment of soil analysis. (a) CHNS elemental analyser for soil total $N$ and $S$ determination. (b) Flame photometer for determination of available potassium in soil. (c) Soil particle size determination by pipet method. (d) Determination of carbonate content in soil by

Scheibler's calcimeter, volumetric method

Based on the analysis of the potential hydrolytic acidity, it was established that the soil on which the experiments were performed belongs to acidic soil. The plots are characterized by a carbonless soil type. The low $\mathrm{pH}$ value and low content of free $\mathrm{CaCO}_{3}$ indicate the possibility of calcium deficiency, which at some stages of plant development may cause reduced availability of magnesium, molybdenum and phosphorus. The soil is medium provided with organic matter and belongs to the class of poorly humus soils. This soil is medium supplied with readily available phosphorus and potassium. The tested soil samples have loam, as well as clay texture and belong to clay loam soil class (Table 1). According to the Ordinance on Control and Certification in Organic Production in Serbia, a maximum intake of $170 \mathrm{~kg} \mathrm{~N}^{-1}$ per year is foreseen for organic agriculture in order to prevent leaching of nitrogen, introduction of heavy metals and harmful organic substances, spread of weeds, pollution of soil by harmful organisms, etc.

\section{Climate data for the tested period}

The production of cultivated plants is highly dependent on climatic factors, primarily rainfall and air temperature, which significantly affect the availability and utilization of 
fertilizers by the plants, and thus the growth and development of the cultivated plants and weeds. We analyzed weather condition (temperature and precipitation) during two year of experiment and during long term period (1992-2010 year) and compared average values of those factors. According to our results, temperature in March and April $2014\left(13.5^{\circ} \mathrm{C}\right.$, $16.3^{\circ} \mathrm{C}$, respectively) was significantly higher than in $2013\left(6.6^{\circ} \mathrm{C}, 13.2^{\circ} \mathrm{C}\right)$ and long term period $\left(7.1^{\circ} \mathrm{C}, 12.0^{\circ} \mathrm{C}\right)$. Other temperatures for growing seasons were similar (Figs. 2 and 3). The meteorological parameters were monitored and registered in the Meteorological station located in Fruit Research Institute, Čačak.

Table 1. Physicochemical analysis of the soil at organic farm

\begin{tabular}{c|c|c|c}
\hline Parameters & Soil under bean & Soil under potato & Average \\
\hline $\mathrm{pH}$ in $\mathrm{KCL}$ & 4.30 & 4.77 & 4.53 \\
$\mathrm{pH}$ in $\mathrm{H}_{2} \mathrm{O}$ & 5.95 & 5.84 & 5.89 \\
Hydrolytic acidity $-\mathrm{H}(\mathrm{meq} / 100 \mathrm{~g})$ & 11.18 & 9.73 & 10.46 \\
$\mathrm{CaCO}_{3}(\%)$ & 0.00 & 0.00 & 0.00 \\
Humus $(\%)$ & 2.91 & 2.50 & 2.70 \\
\hline Content of macronutrients & & & 0.76 \\
Total $\mathrm{N}(\%)^{*}$ & 0.720 & 0.798 & 18.35 \\
Available phosphorus $\mathrm{P}_{2} \mathrm{O}_{5}(\mathrm{mg} / 100 \mathrm{~g})$ & 10.0 & 26.7 & 21.55 \\
Available potassium $\mathrm{K}_{2} \mathrm{O}(\mathrm{mg} / 100 \mathrm{~g})$ & 18.6 & 24.5 & 1.14 \\
\hline Total S $(\%)^{*}$ & 1.05 & 1.24 & 10.44 \\
\hline Organic matter $(\%)$ & & 11.31 & 39.28 \\
\hline Coarse sand $(2-0.2 \mathrm{~mm})$ & 9.57 & 37.89 & 27.04 \\
Fine sand $(0.2-0.02 \mathrm{~mm})$ & 40.67 & 27.52 & 23.24 \\
Powder $(0.02-0.002 \mathrm{~mm})$ & 26.56 & 23.28 & Clay loam \\
\hline Clay $(<0.002 \mathrm{~mm})$ & 23.20 & Clay loam & \\
Texture class & Clay loam & &
\end{tabular}

*Determined by elementary analysis after dry combustion

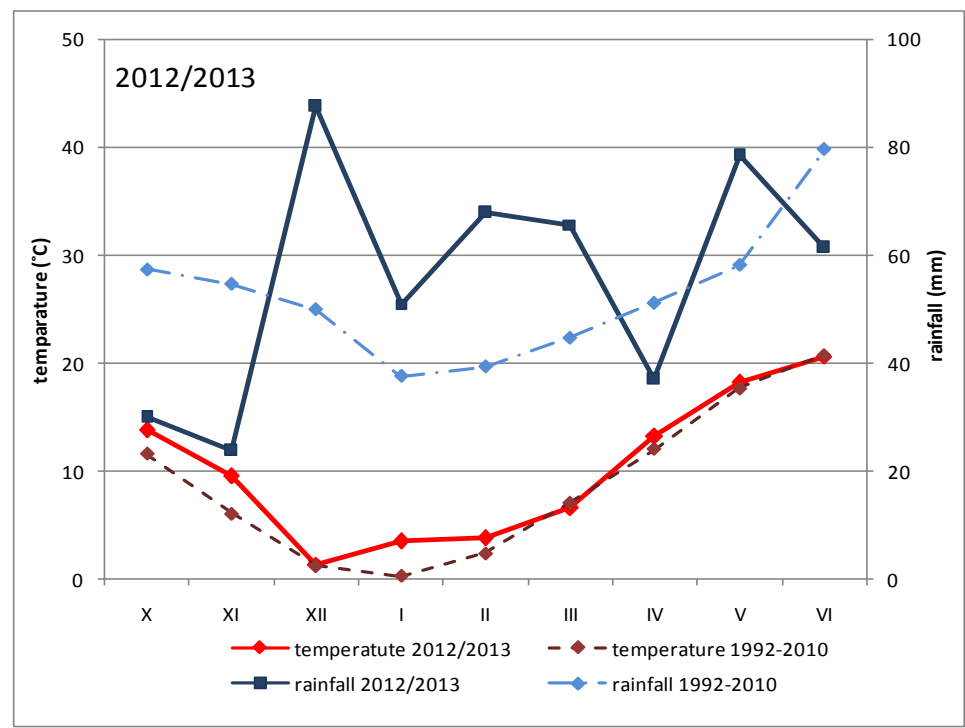

Figure 2. Average temperatures and total rainfall for each month during 2012/2013 growing season and a long-term period (1992-2010) 
Precipitation in January 2014 was only $0.8 \mathrm{~mm}$, which was significantly lower than in $2013(51 \mathrm{~mm})$ and long term period $(37.6 \mathrm{~mm})$. The two growing seasons differed mainly as far as the total rainfall. The total rainfall also differed between two investigated years and long term period. Total precipitation in 2013 was $503 \mathrm{~mm}$, $414 \mathrm{~mm}$ in 2014 and for long term period it was $473 \mathrm{~mm}$. In 2012/2013 rainfall was $30 \mathrm{~mm}$ higher than long term period and $89 \mathrm{~mm}$ higher than 2013. Compared to the multi-year period, in 2013/2014 there was $50 \mathrm{~mm}$ less rain. In the period from October to March 2012/2013, there was $326 \mathrm{~mm}$ rainfall while in the same period of 2013/2014 there was only $95 \mathrm{~mm}$ of rainfall. The 2013/2014 wheat growing season was characterized by high rainfall in May $(167.8 \mathrm{~mm})$ and June $(149.8 \mathrm{~mm})$, which negatively affected plant health and crop maturity. In March 2014 was no precipitation, which has slowed the growth of wheat plants (Fig. 3).

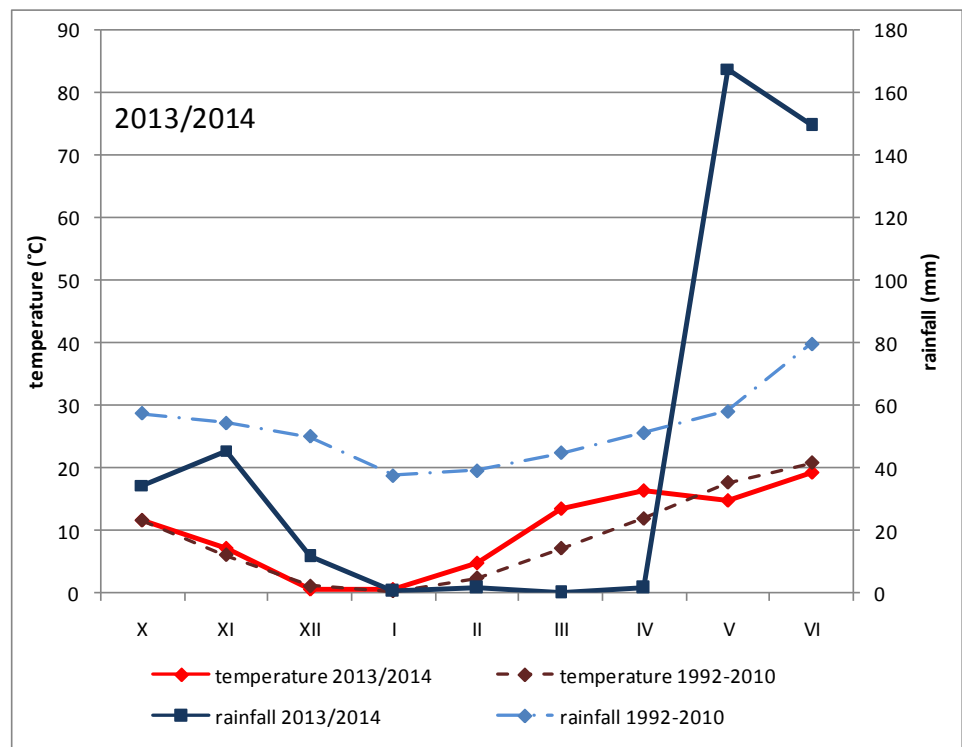

Figure 3. Average temperatures and total rainfall for each month during 2013/2014 growing season and a long-term period (1992-2010)

\section{Statistical data processing}

All the recorded parameters were compared by means of an analysis of variance (ANOVA) according to a random block system with three factors using the MSTAT-C program (Michigan State University, 1990). The significant differences between the average values were estimated by least significant difference test (LSD). Simple correlations between investigated traits were also calculated using MSTAT-C program.

\section{Results}

Mean values for number of spikes $\mathrm{m}^{-2}$ of durum wheat are presented in Table 2. Cultivar Olimpik had the highest average number of spikes per square meter (341.3). Significant lower number of spikes had cultivar Windur (244.9) and genotype KG-443/1 (294.7). The highest values for this component were found with two $\mathrm{N}$ foliar treatments in both investigated year (307.67, 323.86, respectively). In 2014, cultivar Olimpik showed the highest value for number of spikes $\mathrm{m}^{-2}$ in variant with two $\mathrm{N}$ 
treatments (428.0). Nitrogen had a positive effect on the number of spikes $\mathrm{m}^{-2}$. The number of spikes $\mathrm{m}^{-2}$, average for all genotypes and years, on variant with one $\mathrm{N}$ treatment was lower about 3\% than in the control, while on variant with two $\mathrm{N}$ treatments it was higher $14 \%$ than in the control (Table 2).

High significant differences were established between genotypes $(\mathrm{G})$, years $(\mathrm{Y})$ and its interaction $(\mathrm{G} \times \mathrm{Y})$. Applied $\mathrm{N}$ treatment $(\mathrm{T})$ also had high significant influence to number of spikes, interaction between year and applied $\mathrm{N}$ treatment $(\mathrm{Y} \times \mathrm{T})$ was highly significant $(P \leq 0.01)$. Other interactions $(\mathrm{G} \times \mathrm{T}$ and $\mathrm{G} \times \mathrm{Y} \times \mathrm{T})$ were significant $(P \leq 0.05)($ Table 2$)$.

Table 2. Mean values and analysis of variance for number of spikes $m^{-2}$ of durum wheat

\begin{tabular}{|c|c|c|c|c|c|c|c|c|c|}
\hline \multirow{3}{*}{\multicolumn{2}{|c|}{ Genotype }} & \multicolumn{6}{|c|}{ Foliar fertilization } & \multirow{3}{*}{\multicolumn{2}{|c|}{ Average }} \\
\hline & & \multicolumn{2}{|c|}{ T1 } & \multicolumn{2}{|c|}{ T2 } & \multicolumn{2}{|c|}{ T3 } & & \\
\hline & & 2013 & 2014 & 2013 & 2014 & 2013 & 2014 & & \\
\hline \multicolumn{2}{|c|}{ Olimpik } & $411.7^{\mathrm{ab}}$ & $255.7^{\mathrm{f}-\mathrm{m}}$ & $346.3^{\mathrm{a}-\mathrm{e}}$ & $247.0^{\mathrm{i}-\mathrm{m}}$ & $359.0^{\mathrm{a}-\mathrm{d}}$ & $428.0^{\mathrm{a}}$ & \multicolumn{2}{|c|}{341.3} \\
\hline \multicolumn{2}{|c|}{ Windur } & $320.7^{c-j}$ & $236.0^{\mathrm{klm}}$ & $263.0^{\mathrm{f}-\mathrm{l}}$ & $175.0^{\mathrm{m}}$ & $235.3^{\mathrm{lm}}$ & $239.7^{\mathrm{j}-\mathrm{m}}$ & \multicolumn{2}{|c|}{244.9} \\
\hline \multicolumn{2}{|c|}{ Žitka } & $297.0^{c-1}$ & $265.0^{\mathrm{e}-1}$ & $260.3^{\mathrm{f}-\mathrm{l}}$ & $247.3^{\mathrm{i}-\mathrm{m}}$ & $373.7^{\mathrm{abc}}$ & $251.7^{\mathrm{h}-\mathrm{m}}$ & \multicolumn{2}{|c|}{282.5} \\
\hline \multicolumn{2}{|c|}{ KG-28-6 } & $330.3^{\mathrm{b}-\mathrm{h}}$ & $262.7^{f-1}$ & $323.0^{\mathrm{c}-\mathrm{i}}$ & $278.7^{\mathrm{d}-1}$ & $300.7^{\mathrm{c}-1}$ & $338.0^{\mathrm{b}-\mathrm{f}}$ & & 5.6 \\
\hline $\mathrm{KG}$ & $1-3 / 1$ & $253.0^{\mathrm{h}-\mathrm{m}}$ & $242.7^{\mathrm{i}-\mathrm{m}}$ & $318.3^{\mathrm{c}-\mathrm{k}}$ & $311.3^{\mathrm{c}-1}$ & $306.3^{\mathrm{c}-1}$ & $336.3^{\mathrm{b}-\mathrm{g}}$ & & 4.7 \\
\hline $\mathrm{KG}-$ & $-33 / 1$ & $258.3^{\mathrm{f}-1}$ & $248.3^{\mathrm{h}-\mathrm{m}}$ & $256.7^{\mathrm{f}-\mathrm{m}}$ & $262.0^{\mathrm{f}-1}$ & $284.7^{\mathrm{d}-1}$ & $349.3^{\mathrm{a}-\mathrm{d}}$ & & 6.6 \\
\hline KG-? & $05-03$ & $253.7^{\mathrm{h}-\mathrm{m}}$ & $255.7^{\mathrm{f}-\mathrm{m}}$ & $242.3^{\mathrm{i}-\mathrm{m}}$ & $254.0^{\mathrm{g}-\mathrm{m}}$ & $294.0^{\mathrm{c}-1}$ & $324.0^{\mathrm{c}-\mathrm{i}}$ & & 0.6 \\
\hline & age & 303.53 & 252.30 & 287.13 & 253.61 & 307.67 & 323.86 & & 8.02 \\
\hline & & $\mathrm{G}$ & $\mathrm{Y}$ & $\mathrm{G} \times \mathrm{Y}$ & $\mathrm{T}$ & $\mathrm{G} \times \mathrm{T}$ & $\mathrm{Y} \times \mathrm{T}$ & $\mathrm{G} \times$ & $\mathrm{Y} \times \mathrm{T}$ \\
\hline & & 37.87 & - & 53.54 & 43.59 & 58.40 & 61.65 & & .60 \\
\hline & & 57.38 & - & 81.14 & 100.6 & 81.88 & 142.2 & & 5.8 \\
\hline & & & & Analysis & variance & & & & \\
\hline & $\mathrm{G}$ & $\mathrm{Y}$ & $\mathrm{G} \times \mathrm{Y}$ & $\mathrm{T}$ & $\mathrm{G} \times \mathrm{T}$ & $\mathrm{Y} \times \mathrm{T}$ & $\mathrm{G} \times \mathrm{Y} \times \mathrm{T}$ & Error & Total \\
\hline $\mathrm{df}$ & 6 & 1 & 6 & 2 & 12 & 2 & 12 & 82 & 125 \\
\hline MS & 16525.6 & 16457. & 5772.8 & 24844.4 & 4189.9 & 12830.9 & 1443.3 & 2155.7 & - \\
\hline $\mathrm{F}$ & $7.666^{* *}$ & $7.634^{*}$ & $2.678^{* *}$ & $11.525^{\text {** }}$ & $1.944^{*}$ & $5.952^{* *}$ & $1.922^{*}$ & - & - \\
\hline
\end{tabular}

Means followed by different letter(s) within the columns differ significantly at $5 \%$ level of significance

*Significant differences per $P \leq 0.05$. ${ }^{* *}$ Significant differences per $P \leq 0.01$

The number of grains spike $\mathrm{e}^{-1}$ is presented in Table 3. On average for all years and $\mathrm{N}$ treatments, genotype KG-3405-03 had the highest value of number of grains spike ${ }^{-1}$ (48.6). Similar results were shown by cultivar KG-28-6 (48.2). The lowest, significantly different average number of grains spike ${ }^{-1}$ had genotype Olimpik (39.1). On average for all genotypes, in control and both $\mathrm{N}$ foliar treatments, higher number of grains spike ${ }^{-1}$ was established in the second year of investigation. The highest value of this trait, on average, was 2014 in variant with two $\mathrm{N}$ treatments (50.47), and the lowest was 2013 in the control variant (36.71). The number of grains spike ${ }^{-1}$, on average for all genotypes and years, after spraying were increased about $16 \%$ (variant with one $\mathrm{N}$ treatment), and $28 \%$ (variant with two $\mathrm{N}$ treatments) higher than in the control.

High significant differences $(P \leq 0.01)$ were established between genotypes $(\mathrm{G})$, years $(\mathrm{Y})$, applied $\mathrm{N}$ treatment $(\mathrm{T})$ and its interactions, except interactions $\mathrm{Y} \times \mathrm{T}$ which was not significant (Table 3). In this investigation, genotypes had different response to foliar nutrition in both year of investigation. All the treatments resulted in a significantly more grains spike ${ }^{-1}$ than the check plots did. 


$$
-178-
$$

Table 3. Mean values and analysis of variance for number of grains per spike

\begin{tabular}{|c|c|c|c|c|c|c|c|c|c|}
\hline \multirow{3}{*}{\multicolumn{2}{|c|}{ Genotype }} & \multicolumn{6}{|c|}{ Foliar fertilization } & \multirow{3}{*}{\multicolumn{2}{|c|}{ Average }} \\
\hline & & \multicolumn{2}{|c|}{ T1 } & \multicolumn{2}{|c|}{$\mathrm{T} 2$} & \multicolumn{2}{|r|}{ T3 } & & \\
\hline & & 2013 & 2014 & 2013 & 2014 & 2013 & 2014 & & \\
\hline \multicolumn{2}{|c|}{ Olimpik } & $31.33^{\mathrm{uv}}$ & $39.33^{\text {qrs }}$ & $35.0^{\mathrm{t}}$ & $41.0^{\mathrm{opq}}$ & $44.33^{\mathrm{jkl}}$ & $43.33^{\mathrm{kln}}$ & & .1 \\
\hline \multicolumn{2}{|c|}{ Windur } & $40.0^{\mathrm{pqr}}$ & $38.0^{\mathrm{s}}$ & $45.0^{\mathrm{jk}}$ & $43.0^{1 \mathrm{mn}}$ & $48.0^{\mathrm{gh}}$ & $47.0^{\mathrm{hi}}$ & & 3.5 \\
\hline \multicolumn{2}{|c|}{ Žitka } & $40.0^{\mathrm{pqr}}$ & $39.33^{\mathrm{qrs}}$ & $41.0^{\mathrm{opq}}$ & $45.0^{\mathrm{jk}}$ & $46.0^{\mathrm{ij}}$ & $47.67^{\mathrm{gh}}$ & & 3.2 \\
\hline \multicolumn{2}{|c|}{ KG-28-6 } & $40.33^{\mathrm{o}-\mathrm{r}}$ & $43.0^{\mathrm{lmn}}$ & $47.33^{\mathrm{hi}}$ & $49.33^{\mathrm{fg}}$ & $53.0^{\mathrm{cd}}$ & $56.0^{\mathrm{b}}$ & & .2 \\
\hline \multicolumn{2}{|c|}{ KG-44-3/1 } & $29.67^{v}$ & $44.67^{\mathrm{jkl}}$ & $39.0^{\mathrm{rs}}$ & $51.0^{\mathrm{ef}}$ & $42.0^{\mathrm{mno}}$ & $54.33^{\mathrm{bc}}$ & & .4 \\
\hline \multicolumn{2}{|c|}{ KG-43-33/1 } & $31.67^{\mathrm{u}}$ & $39.0^{\mathrm{rs}}$ & $41.33^{\text {nop }}$ & $44.33^{\mathrm{jkl}}$ & $46.0^{\mathrm{ij}}$ & $53.33^{\mathrm{cd}}$ & & 2.6 \\
\hline \multicolumn{2}{|c|}{ KG-3405-03 } & $44.0^{\mathrm{kl}}$ & $37.67^{s}$ & $56.0^{\mathrm{b}}$ & $44.0^{\mathrm{kl}}$ & $58.33^{\mathrm{a}}$ & $51.67^{\mathrm{de}}$ & & 3.6 \\
\hline \multicolumn{2}{|c|}{ Average } & 36.71 & 40.14 & 43.52 & 45.38 & 48.24 & 50.47 & & .01 \\
\hline \multicolumn{2}{|c|}{ LSD } & $\mathrm{G}$ & $\mathrm{Y}$ & $\mathrm{G} \times \mathrm{Y}$ & $\mathrm{T}$ & $\mathrm{G} \times \mathrm{T}$ & $\mathrm{Y} \times \mathrm{T}$ & & $Y \times T$ \\
\hline \multicolumn{2}{|c|}{0.05} & 1.736 & - & 2.456 & 1.999 & 2.074 & NS & & 753 \\
\hline \multicolumn{2}{|c|}{0.01} & 2.631 & - & 3.721 & 4.611 & 2.908 & NS & & 58 \\
\hline \multicolumn{10}{|c|}{ Analysis of variance } \\
\hline & $\mathrm{G}$ & $\mathrm{Y}$ & $\mathrm{G} \times \mathrm{Y}$ & $\mathrm{T}$ & $\mathrm{G} \times \mathrm{T}$ & $\mathrm{Y} \times \mathrm{T}$ & $\mathrm{G} \times \mathrm{Y} \times \mathrm{T}$ & Error & Total \\
\hline $\mathrm{df}$ & 6 & 1 & 6 & 2 & 12 & 2 & 12 & 82 & 125 \\
\hline MS & 198.63 & 198.13 & 197.15 & 1256.44 & 16.55 & 7.06 & 10.68 & 4.53 & - \\
\hline $\mathrm{F}$ & $43.824^{* *}$ & $43.714^{*}$ & $43.498^{* *}$ & $277.658^{* *}$ & $3.651^{* *}$ & $1.557^{\mathrm{ns}}$ & $2.358^{* *}$ & - & - \\
\hline
\end{tabular}

Means followed by different letter(s) within the columns differ significantly at $5 \%$ level of significance

${ }^{*}$ Significant differences per $P \leq 0.05$. ${ }^{* *}$ Significant differences per $P \leq 0.01$. ${ }^{\text {ns }}$ Non significant

Genotype KG-3405-03, on average, had the highest value of TGW (40.36 g), while Olimpik genotype had the lowest $(35.44 \mathrm{~g})$. On average for all genotypes, the highest TGW was in 2013 on variant with two $\mathrm{N}$ treatments $(45.24 \mathrm{~g})$, and the lowest was in 2014 on control variant (32.72 g) (Table 4).

On average for all variants and genotypes, higher TGW was established in the first investigated year compared to the second year. TGW, on average for all genotypes and years, after spraying were up 7\% (variant with one $\mathrm{N}$ treatment), and 13\% (variant with two $\mathrm{N}$ treatments) higher than in the control. Thousand grains weight highly depended of genotype, year, $N$ treatment and interactions $G \times Y$ and $Y \times T$. Interactions $G \times T$ and $\mathrm{G} \times \mathrm{Y} \times \mathrm{T}$ were not significant for $\mathrm{TGW}$. Year had the highest influence on $\mathrm{TGW}$ (Table 4).

The tested durum genotypes on average did not significantly differ in regards to grain yield (Table 5). Cultivar Olimpik, on average for all treatments, had the highest grain yield $\left(178.4 \mathrm{~g} \mathrm{~m}^{-2}\right)$, and $\mathrm{KG}-28-6$ had the lowest yield $\left(159.8 \mathrm{~g} \mathrm{~m}^{-2}\right)$. On average for all $\mathrm{N}$ variants and genotypes, it was established that grain yield in the first year of experiment was higher than in the second year. The highest average value of grain yield of all genotypes was established in the variant with two $\mathrm{N}$ treatments in 2013 year $\left(221.5 \mathrm{~g} \mathrm{~m}^{-2}\right)$. The lowest grain yield $\left(128.6 \mathrm{~g} \mathrm{~m}^{-2}\right)$ was found on the control variant in 2014. Grain yield, on average for all genotypes and years after spraying, was increased for $22 \%$ (one $\mathrm{N}$ treatment), and $54 \%$ (two $\mathrm{N}$ treatments) were higher than in the control. Also, the grain yield on average for all genotypes and years on variant with two $\mathrm{N}$ treatments was higher for about $26 \%$ than on variant with one $\mathrm{N}$ treatment. 


$$
-179-
$$

Table 4. Mean values and analysis of variance for TGW of durum wheat ( $g$ )

\begin{tabular}{|c|c|c|c|c|c|c|c|c|c|}
\hline \multirow{3}{*}{\multicolumn{2}{|c|}{ Genotype }} & \multicolumn{6}{|c|}{ Foliar fertilization } & \multirow{3}{*}{\multicolumn{2}{|c|}{ Average }} \\
\hline & & \multicolumn{2}{|c|}{ T1 } & \multicolumn{2}{|c|}{$\mathrm{T} 2$} & \multicolumn{2}{|r|}{ T3 } & & \\
\hline & & 2013 & 2014 & 2013 & 2014 & 2013 & 2014 & & \\
\hline \multicolumn{2}{|c|}{ Olimpik } & $34.13^{\mathrm{h}-\mathrm{k}}$ & $33.27^{\mathrm{ijk}}$ & $35.20^{f-j}$ & $33.47^{\mathrm{ijk}}$ & $40.83^{\mathrm{b}-\mathrm{h}}$ & $35.73^{\mathrm{f}-\mathrm{j}}$ & & 35.44 \\
\hline \multicolumn{2}{|c|}{ Windur } & $42.93^{\mathrm{a}-\mathrm{e}}$ & $34.93^{\mathrm{f}-\mathrm{j}}$ & $43.00^{\mathrm{a}-\mathrm{e}}$ & $36.07^{e-j}$ & $48.43^{\mathrm{a}}$ & $33.57^{\mathrm{ijk}}$ & & 39.82 \\
\hline \multicolumn{2}{|c|}{ Žitka } & $39.43^{\mathrm{c}-\mathrm{i}}$ & $27.40^{\mathrm{k}}$ & $40.90^{\mathrm{b}-\mathrm{h}}$ & $32.83^{\mathrm{ijk}}$ & $43.70^{\mathrm{a}-\mathrm{c}}$ & $32.80^{\mathrm{ijk}}$ & & 36.18 \\
\hline \multicolumn{2}{|c|}{ KG-28-6 } & $37.80^{\mathrm{d}-\mathrm{j}}$ & $33.47^{\mathrm{ijk}}$ & $41.40^{\mathrm{a}-\mathrm{g}}$ & $33.60^{\mathrm{ijk}}$ & $48.43^{\mathrm{a}}$ & $35.60^{f-j}$ & & 38.38 \\
\hline \multicolumn{2}{|c|}{ KG-44-3/1 } & $35.27^{\mathrm{f}-\mathrm{j}}$ & $34.40^{\mathrm{g}-\mathrm{k}}$ & $38.73^{\mathrm{d}-\mathrm{j}}$ & $35.40^{\mathrm{f}-\mathrm{j}}$ & $41.83^{\mathrm{a}-\mathrm{f}}$ & $36.80^{\mathrm{d}-\mathrm{j}}$ & & 37.07 \\
\hline \multicolumn{2}{|c|}{ KG-43-33/1 } & $37.20^{\mathrm{d}-\mathrm{j}}$ & $32.23^{\mathrm{jk}}$ & $43.30^{\mathrm{a}-\mathrm{d}}$ & $34.60^{\mathrm{g}-\mathrm{j}}$ & $47.07^{\mathrm{ab}}$ & $34.70^{\mathrm{g}-\mathrm{j}}$ & & 38.18 \\
\hline \multicolumn{2}{|c|}{ KG-3405-03 } & $40.97^{\mathrm{b}-\mathrm{h}}$ & $33.33^{\mathrm{ijk}}$ & $45.90^{\mathrm{abc}}$ & $37.53^{\mathrm{d}-\mathrm{j}}$ & $46.43^{\mathrm{abc}}$ & $38.00^{\mathrm{d}-\mathrm{j}}$ & & 40.36 \\
\hline \multicolumn{2}{|c|}{ Average } & 38.25 & 32.72 & 41.20 & 34.78 & 45.24 & 35.31 & & 37.92 \\
\hline \multicolumn{2}{|c|}{ LSD } & $\mathrm{G}$ & $\mathrm{Y}$ & $G \times Y$ & $\mathrm{~T}$ & $\mathrm{G} \times \mathrm{T}$ & $\mathrm{Y} \times \mathrm{T}$ & & $\mathrm{G} \times \mathrm{Y} \times \mathrm{T}$ \\
\hline \multicolumn{2}{|c|}{0.05} & 3.238 & - & 4.580 & 3.728 & NS & 5.272 & & NS \\
\hline \multicolumn{2}{|c|}{0.01} & 4.906 & - & 6.939 & 8.599 & NS & 12.160 & & NS \\
\hline \multicolumn{10}{|c|}{ Analysis of variance } \\
\hline & $\mathrm{G}$ & $\mathrm{Y}$ & $G \times Y$ & $\mathrm{~T}$ & $\mathrm{G} \times \mathrm{T}$ & $\mathrm{Y} \times \mathrm{T}$ & $\mathrm{G} \times \mathrm{Y} \times \mathrm{T}$ & Error & Total \\
\hline $\mathrm{df}$ & 6 & 1 & 6 & 2 & 12 & 2 & 12 & 82 & 125 \\
\hline MS & 59.31 & 1675.72 & 45.02 & 241.86 & 6.73 & 56.95 & 6.57 & 15.72 & - \\
\hline $\mathrm{F}$ & $3.772^{* *}$ & $106.574^{* *}$ & $2.863^{*}$ & $15.382^{* *}$ & $0.428^{\mathrm{ns}}$ & $3.622^{*}$ & $0.418^{\mathrm{ns}}$ & - & - \\
\hline
\end{tabular}

Means followed by different letter(s) within the columns differ significantly at $5 \%$ level of significance

*Significant differences per $P \leq 0.05$. ${ }^{* *}$ Significant differences per $P \leq 0.01$; ${ }^{\text {ns }}$ Not significant

Table 5. Mean values and analysis of variance for grain yield of durum wheat $\left(\mathrm{g} \mathrm{m}^{-2}\right)$

\begin{tabular}{|c|c|c|c|c|c|c|c|c|c|c|c|}
\hline \multirow{3}{*}{\multicolumn{2}{|c|}{ Genotype }} & \multicolumn{8}{|c|}{ Foliar fertilization } & \multirow{3}{*}{\multicolumn{2}{|c|}{ Average }} \\
\hline & & \multicolumn{3}{|c|}{ T1 } & \multicolumn{3}{|c|}{$\mathbf{T 2}$} & \multicolumn{2}{|c|}{ T3 } & & \\
\hline & & 2013 & \multicolumn{2}{|c|}{2014} & 2013 & \multicolumn{2}{|c|}{2014} & 2013 & 2014 & & \\
\hline \multicolumn{2}{|c|}{ Olimpik } & $133.8^{1-0}$ & \multicolumn{2}{|c|}{$116.3^{\circ}$} & $190.3^{\mathrm{c}-\mathrm{h}}$ & \multicolumn{2}{|c|}{$147.00^{\mathrm{i}-\mathrm{o}}$} & $253.0^{\mathrm{a}}$ & $229.8^{\mathrm{abc}}$ & \multicolumn{2}{|c|}{178.4} \\
\hline \multicolumn{2}{|c|}{ Windur } & $153.1^{\mathrm{q}-\mathrm{o}}$ & \multicolumn{2}{|c|}{$136.7^{1-\mathrm{o}}$} & $156.0^{\mathrm{f}-\mathrm{o}}$ & \multicolumn{2}{|c|}{$149.3^{\mathrm{h}-\mathrm{o}}$} & $219.3^{\mathrm{a}-\mathrm{d}}$ & $198.7^{\mathrm{b}-\mathrm{e}}$ & \multicolumn{2}{|c|}{168.8} \\
\hline \multicolumn{2}{|c|}{ Žitka } & $140.0^{\mathrm{k}-\mathrm{o}}$ & \multicolumn{2}{|c|}{$130.0^{\mathrm{mno}}$} & $174.7^{\mathrm{e}-\mathrm{l}}$ & \multicolumn{2}{|c|}{$151.5^{\mathrm{g}-\mathrm{o}}$} & $187.9^{\mathrm{d}-\mathrm{i}}$ & $182.7^{\mathrm{d}-\mathrm{j}}$ & \multicolumn{2}{|c|}{161.1} \\
\hline \multicolumn{2}{|c|}{ KG-28-6 } & $139.8^{\mathrm{k}-\mathrm{o}}$ & \multicolumn{2}{|c|}{$127.3^{\text {n-o }}$} & $170.2^{\mathrm{e}-\mathrm{m}}$ & \multicolumn{2}{|c|}{$143.5^{\mathrm{j}-\mathrm{o}}$} & $192.1^{\mathrm{c}-\mathrm{g}}$ & $186.0^{\mathrm{d}-\mathrm{i}}$ & \multicolumn{2}{|c|}{159.8} \\
\hline \multicolumn{2}{|c|}{ KG-44-3/1 } & $131.2^{\mathrm{mno}}$ & \multicolumn{2}{|c|}{$130.7^{\mathrm{mno}}$} & $192.3^{\mathrm{c}-\mathrm{g}}$ & \multicolumn{2}{|c|}{$150.2^{\mathrm{h}-\mathrm{o}}$} & $259.7^{\mathrm{a}}$ & $190.4^{\mathrm{c}-\mathrm{h}}$ & \multicolumn{2}{|c|}{175.7} \\
\hline \multicolumn{2}{|c|}{ KG-43-33/1 } & $135.6^{1-o}$ & \multicolumn{2}{|c|}{$129.7^{\mathrm{mno}}$} & $196.0^{\mathrm{b}-\mathrm{f}}$ & \multicolumn{2}{|c|}{$140.9^{\mathrm{k}-\mathrm{o}}$} & $236.0^{\mathrm{ab}}$ & $180.5^{\mathrm{d}-\mathrm{k}}$ & \multicolumn{2}{|c|}{169.8} \\
\hline KG-3 & $05-03$ & $137.1^{1-0}$ & & $7^{\mathrm{mno}}$ & $173.2^{\mathrm{e}-1}$ & & $5.0^{\mathrm{f}-\mathrm{o}}$ & $202.4^{\mathrm{b}-\mathrm{e}}$ & $167.0^{e-n}$ & & 0.7 \\
\hline Av & age & 138.7 & & 8.6 & 179.0 & & 48.2 & 221.5 & 190.7 & & .78 \\
\hline & & $\mathrm{G}$ & & $l$ & $G \times Y$ & & $\mathrm{~T}$ & $\mathrm{G} \times \mathrm{T}$ & $\mathrm{Y} \times \mathrm{T}$ & $\mathrm{G} \times$ & $I \times \mathrm{T}$ \\
\hline & & NS & & 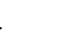 & NS & & 1.90 & 29.34 & NS & & S \\
\hline & & NS & & 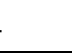 & NS & & 0.52 & 41.13 & NS & & S \\
\hline & & & & & Analysis & & ance & & & & \\
\hline & $\mathrm{G}$ & $\mathrm{Y}$ & & $G \times$ & $\mathrm{T}$ & & $\mathrm{G} \times \mathrm{T}$ & $\mathrm{Y} \times \mathrm{T}$ & $\mathrm{G} \times \mathrm{Y} \times \mathrm{T}$ & Error & Total \\
\hline df & 6 & 1 & & 6 & 2 & & 12 & 2 & 12 & 82 & 125 \\
\hline MS & 1013.65 & 17935 & & 539. & 55687 & & 1133.80 & 1503.86 & 410.49 & 544.05 & - \\
\hline $\mathrm{F}$ & $1.863^{\mathrm{ns}}$ & 32.96 & & 0.99 & 102.3 & & $2.084^{*}$ & $2.764^{\mathrm{ns}}$ & $0.754^{\mathrm{ns}}$ & - & - \\
\hline
\end{tabular}

Means followed by different letter(s) within the columns differ significantly at $5 \%$ level of significance

${ }^{*}$ Significant differences per $P \leq 0.05$. ${ }^{* *}$ Significant differences per $P \leq 0.01$. ${ }^{\text {ns}}$ Non significant 
Significant differences were found between tested years, $N$ treatments and $G \times T$ interaction for grain yield. The differences between genotypes and other interactions $(\mathrm{G} \times \mathrm{Y}, \mathrm{Y} \times \mathrm{T}$ and $\mathrm{G} \times \mathrm{Y} \times \mathrm{T})$ were not statistically significant (Table 5). Treatment and year had the highest influence to grain yield $\left(\mathrm{F}=102.357^{* *} ; 32.967^{* *}\right.$, respectively).

The correlations between the studied yield components and grain yield of durum wheat were analyzed (Table 6). Grain yield positively correlated to the other traits (TGW, number of spikes $\mathrm{m}^{-2}$ and number of grains spike ${ }^{-1}$ ), which were statistically highly significant. The highest correlation was between yield and TGW $\left(0.501^{* *}\right)$. The correlation between number of grains spike ${ }^{-1}$ and TGW $\left(0.288^{* *}\right)$, were also positive and statistically highly significant.

Table 6. Correlations between yield and other investigated parameters of durum wheat

\begin{tabular}{|c|c|c|c|}
\hline Components & TGW & Number of spikes $\mathbf{m}^{-2}$ & Number of grains spike ${ }^{-1}$ \\
\hline Yield & $0.501 * *$ & $0.339 * *$ & $0.333^{* *}$ \\
\hline TGW & & $0.039^{\mathrm{ns}}$ & $0.288 * *$ \\
\hline Number of spikes $\mathrm{m}^{-2}$ & & & $0.055^{\mathrm{ns}}$ \\
\hline Number of grains spike ${ }^{-1}$ & & & - \\
\hline
\end{tabular}

**Significant differences per $P \leq 0.01$. ${ }^{\text {ns Not significant }}$

\section{Discussion}

Agricultural land is, as a rule, scarce in nitrogen. This is the reason that it is necessary to add fertilizer with the appropriate nitrogen content, which is an important mineral element in growing crops and achieving higher yields and quality. The effect of nitrogen nutrition on increasing wheat grain yield depends on the dose of nitrogen and the time and manner of its application (Tedone et al., 2017; Dolijanović et al., 2019). The application of nitrogen fertilizer must be appropriate for each genotype, which differ in their capacity for $\mathrm{N}$ absorption, translocation and reutilization. Excessive addition of nitrogen causes the lodging of plants, which creates favorable conditions for the development of diseases, weeds, less capacity and competition of lodged plants for the use of mineral nutrients, water, light (Mascianica and Valden, 1986; Bruckner and Morey, 1988; Zhang et al., 2017).

Generally, according to our results, the nitrogen foliar application had a positive effect on the number of spikes $\mathrm{m}^{-2}$, number of grains spikes ${ }^{-1}$, TGW and grain yield, which is in line with other studies (Arif et al., 2006; Khan et al., 2009; Tedone et al., 2014; Arshadullah et al., 2015), who also found a positive effect of nitrogen on these properties. Blandino et al. (2015) have established a significant effect of $\mathrm{N}$ fertilization at the time of late stage of plant development on grain yield in durum wheat, as well as in common wheat (Khan et al., 2009). Galieni et al. (2016) also established that $\mathrm{N}$ fertilization had a significant effect on yield in durum wheat. In our results, it was established that grain yield of genotypes is significantly affected by the $\mathrm{N}$ rate, year and its interactions, which was similar to another research in durum wheat (Ayadi et al., 2014).

Our investigation found that the two growing seasons greatly differed in the amount and distribution of rainfall. Climatic conditions during 2012/2013 were characterized by even distribution of precipitation during the vegetative and generative development of the examined genotypes. In 2013/2014 a significant lack of precipitation was recorded 
(December - April) during the vegetative development of the examined genotypes. On the other hand, large amounts of rainfall were recorded at the time of heading, grain filling and maturation (Maj - June), favored the development of fungal diseases of spikes and grains, which affected yield components and formation of yield. Overall, the mean values of grain yield, number of spikes $\mathrm{m}^{-2}$ and TGW of wheat genotypes were higher in the more favorable 2013/2014 than in the unfavorable 2013/2014 growing season.

Observed by years and applied treatments, a specific reaction of genotypes was recorded in the examined traits. For the number of spikes $\mathrm{m}^{-2}$, the application of different treatments of foliar application of nitrogen in the cultivar Windur did not affect the increase of the average values of the observed trait. In the variety Žitka, an increased number of spikes $\mathrm{m}^{-2}$ was determined only during 2012/2013, considering the favorable climatic conditions in the phase of vegetative development. The variety Olimpik and breeding lines selected at the Small Grains Research Centre in Kragujevac expressed wide adaptability and stability and thus the predominant influence of genotype in the formation of the examined trait, regardless of climatic conditions in the years of testing. Similarly, the high adaptability and stability of bread wheat genotypes selected at the Center from Kragujevac were found in research by Luković et al. (2020) in years with unfavorable climatic conditions.

For the number of grains per spike, all tested genotypes responded positively to foliar application of nitrogen, especially in T3 treatment. It can be concluded that the application of nitrogen and amino acids in the heading and anthesis phase had a positive effect on reducing stress in the examined genotypes due to excessive rainfall. However, the number of grains per spike was on average higher in the 2013/2014 growing season, but due to unfavorable conditions during the stage of grain filling the grains were underdeveloped and small, which had a negative impact on yield. Drought in the early stages of development can seriously affect the number of plants per square meter, and in the tillering phase affects the number of shoots per plant, while drought during stem elongation influenced decreasing of plant height. Later, drought in the phases of flowering, fertilization and grain formation significantly affects the number of grains per area. In the stage of forming grains, drought significantly affects the process of translocation of assimilates to the grain, which had impact on reducing of weight of grains (Sarto et al., 2017).

In contrast to the previously examined traits, a more significant impact of the year was recorded for TGW, which is reflected in lower average values during 2013/2014 for all applied treatments compared to 2012/2013.

Excessive precipitation recorded during heading and anthesis in 2013/2014 influenced that examined genotypes achieved lower average grain yields for all observed treatments compared to 2012/2013. The lower average values of TGW in the tested genotypes, determined during 2013/2014 vegetation season that characterized with excessive amount of precipitation. In this season there was a high degree of correlation between TGW and grain yield. The results of the research indicate that climatic conditions during the year of testing have a decisive influence on the efficiency of foliar application of $\mathrm{N}$ in the phases of heading and anthesis and the formation of the final grain yield in durum wheat genotypes.

Previous research (Hirzel et al., 2010) has shown that splitting the application of nitrogen into three periods (sowing, tillering, stem elongation), appears more efficient than just one application, producing an increase of $15 \%$, or twice, where the increase is 
7\% (Tedone et al., 2014). This strategy appears to be effective in reducing the loss of soil nitrates, which is more dangerous during the winter period, as reported from several authors, due to the rainfall (López-Bellido, 2005). Foliar fertilization is necessary and recommended as a measure that can reduce plant stress due to adverse climatic conditions: high or low air temperature, drought, short or long-term water shortages, etc. (Doflerus, 2014; Trnka et al., 2014; Knežević et al., 2019). In conventional production, where the nutrition of plants is balanced based on the quality of the soil, foliar fertilization achieves a smaller effect. In organic production, plants are more exposed to stressful conditions, because the application of mineral fertilizers and plant protection products is restricted or prohibited, foliar application of nutrients gives better results. The yield of durum wheat with organic cropping system was $21 \%$ lower than in conventional based on the findings of the researchers Fagnano et al. (2012).

Some authors, with foliar fertilizer applications have found increases in grain yield in some years, but on average of five years, foliar fertilizers did not increase grain yield (Staugaitis et al., 2017). Zečević et al. (2004) reported increasing of grain yield and quality under $\mathrm{N}$ applications in flowering and milky stages. Woolfolk et al. (2002) suggested that foliar $\mathrm{N}$ applications before or immediately after flowering may significantly increase grain protein in winter wheat. Managing $\mathrm{N}$ fertilizer is difficult because it depends on many terms such as fertilizer source, quantity, mode of application, application time, variety response and climatic conditions. In this respect, the grain filling period is crucial for the quality of the durum wheat (Orcen et al., 2013; Branković et al., 2015b).

The application of fertilizers in system of organic production has the function of increasing the fertility and biological activity of the soil and at the same time providing the nutrients necessary for the growth and development of the plants. In organic system of production, the basic principles are increasing of organic matter in soil and production of healthy food. In order to allow efficient use of nitrogen by plants, when determining the required quantities of organic matter and right time of introduction into the soil, it is necessary to consider all factors that affect the dynamics and balance of nitrogen in the soil, and thus the availability of nitrogen for plants. The results obtained in our investigation were compared with those in the earlier reports (Gopinath et al., 2008), which found that grain yields increased in response to increasing application rates of organic amendments. Dolijanović et al. (2017) found a significant effect of organic and microbiological fertilizers on grain yield in durum, spelt and bread wheat varieties growing in system of organic productions.

Đokić (1988) points out that when wheat is forming grain yield, it is possible to compensate $\mathrm{N}$ deficiency through certain components of the yield. Therefore, if the improved nitrogen nutrition did not sufficiently reflect on tillering, the $\mathrm{N}$ deficiency would be compensated by increasing the number of grains per spike. In case the grain number does not increase, the grain mass will be increased.

According to our study, the grain yield was positively correlated with other traits (TGW, number of spike $\mathrm{m}^{-2}$ and number of grains spike ${ }^{-1}$ ), which were statistically highly significant. These results are in agreement with studies of Khan et al. (2013), Matković et al. (2018) and Nofouzi (2018) who also established positive correlations between yield and yield components in durum wheat. García del Moral et al. (2003) have also found that number of spikes per square meter, TGW and number of kernels/spike were positively related with grain yield. Lupini et al. (2021) have found high positive correlation (0.97) between grain yield and nitrogen use efficiency of 
durum wheat. Investigation by Mariem et al. (2020) had shown that nitrogen fertilization applied from anthesis to maturity had small effects on durum wheat grain yield but had a major impact on grain quality.

\section{Conclusion}

Nitrogen nutrition is one of the main factors that leads to increased productivity and improved quality of wheat grain. In this study, foliar application of nitrogen in heading and anthesis caused an increase in grain yield and the yield components tested.

Number of spikes per square meter, number of grains $\mathrm{m}^{-2}$, thousand grain weight, and wheat yield were significantly increased $(\mathrm{P} \leq 0.01)$ by different levels of fertilizers. The foliar fertilization had a significant effect on yield in both growing seasons. Grain yield, on average for all genotypes and years, after spraying were $22 \%$ (one $\mathrm{N}$ treatment), and 54\% (two $\mathrm{N}$ treatments) higher than in the control. Grain yield was higher for about $26 \%$ in variant with two $\mathrm{N}$ treatments than in variant with one $\mathrm{N}$ treatment.

Grain yield was positively correlated with other traits (TGW, number of spike $\mathrm{m}^{-2}$ and number of grains spike $^{-1}$ ), which were statistically highly significant. The difference in grain yield was found between the investigated genotypes of durum wheat by individual years, but on average for both years, the genotypes responded similarly to the applied foliar nitrogen nutrition. The tested genotypes showed significantly lower yields compared to their genetic potential because durum wheat has low frost-tolerance and during the winter period some of the plants die and the potential for grain yield decreases.

The conducted research indicates that the foliar application of nitrogen and amino acids in the most important stages of plant development (heading and anthesis) is economically justified, especially in years with optimal conditions, considering the established statistical significance of the examined traits.

The significant impact in achievement of potential yield of wheat genotype as well as other cultivated plants, have soil biological and phisico-chemical properties, which can change and modified under scientific farming measures practices: tillage, fertilizing, land reclamation to enhance soil fertility, creation and maintenance of optimal water-air regime of the soil, in order to provide favorable conditions for the growth and development of wheat cultivation and to achieve stable yields. The further investigation is necessary to be done to establish the optimum fertilizer combination to improved crop production, as well as application of nitrogen and amino acids in the grain feeling phenophase on the final yield and technological grain quality of durum wheat.

Acknowledgements. This study was supported by the Ministry of Education, Science and Technological Development of the Republic of Serbia (Grant No. 451-03-9/2021-14/200216).

\section{REFERENCES}

[1] AOAC 972.43 (2000): Method 972.43. Micro-chemical Determination of Carbon, Hydrogen, and Nitrogen, Automated Method. Official Methods of Analysis of AOAC International. 17th Ed. - AOAC International, Arlington, VA.

[2] Araus, J. L., Slafer, M. P., Reynolds, M. P., Royo, C. (2002): Plant breeding and drought in C3 cereals: what should we breed for? - Annals of Botany 89(7): 925-940. DOI: 10.1093/aob/mcf049. 
[3] Arif, M., Chohan, M. A., Ali, S., Gul, R., Khan, S. (2006): Response of wheat to foliar application of nutrients. - Journal of Agricultural and Biological Science 1(4): 30-34.

[4] Arshadullah, M., Ali, A., Hyder, S. I., Mahmood, I. A., Zaman, B. (2015): Effect of $\mathrm{K}_{2} \mathrm{SO}_{4}$ and $\mathrm{KNO}_{3}$ foliar application on wheat growth. - Pakistan Journal of Science and Industrial Researsh Series B: Biological Science 58(1): 19-22.

[5] Ayadi, S., Karmous, C., Hammami, Z., Trifa, Y., Rezgui, S. (2014): Variation of durum wheat yield and nitrogen use efficiency under Mediterranean rainfed environment. International Journal of Agriculture and Crop Sciences 7(10): 693-699.

[6] Blandino, M., Vaccino, P., Reyneri, A. (2015): Late-season nitrogen increases improver common and durum wheat quality. - Agronomy Journal 107: 680-690. DOI: 10.2134/agronj14.0405.

[7] Bošković, J., Hojka, Z., Zečević, V., Prodanović, R., Vukić, M. (2016): Significant resources of sustainable agriculture and organic food production system in Serbia. - Acta Agriculturae Serbica 21(41): 65-85.

[8] Branković, G., Dragičević, V., Dodig, D., Zorić, M., Knežević, D., Žilić, S., Denčić, S., Šurlan, G. (2015a): Genotype $\times$ Environment interaction for antioxidants and phytic acid contents in bread and durum wheat as influenced by climate. - Chilean Journal of Agricultural Research 75(2): 139-146.

[9] Branković, G., Dragičević, V., Dodig, D., Knežević, D., Kobiljski, B., Šurlan-Momirović, G. (2015b): Albumin content in bread wheat (Triticum aestivum L.) and durum wheat (Triticum durum Desf.) as affected by environment. - Scientific Journal Zemdirbyste Agriculture 102(3): 281-288.

[10] Branković, G., Dragičević, V., Žilić, S., Knežević, D., Đurić, N., Dodig, D. (2016): Expected genetic advance and stability of phytic acid and antioxidants content in bread and durum wheat. - Genetika 48(3): 867-880.

[11] Branković, G., Dodig, D., Pajić, V., Kandić, V., Knežević, D., Đurić, N., Živanović, T (2018): Genetic parameters of Triticum aestivum and Triticum durum for technological quality properties in Serbia. - Zemdirbyste - Agriculture 105(1): 39-48.

[12] Bruckner, P. L., Morey, D. D. (1988): Nitrogen effects on soft red winter wheat yield. Agronomic characteristics and quality. - Crop Science 28: 152-157.

[13] Doflerus, R. (2014): To grow or not to grow: a stressful decision for plants. - Plant Science 229: 247-261. https://doi.org/10.1016/j.plantsci.2014.10.002.

[14] Dolijanović, Ž., Kovačević, D., Oljača, S., Roljević Nikolić, S., Šeremešić, S. (2017): Effect of fertilizers on the yield of alternative small grains. - Contemporary Agriculture 66(3-4): 15-21. DOI: 10.1515/contagri-2017-0014.

[15] Dolijanović, Ž., Roljević Nikolić, S., Kovačević, D., Djurdjić, S., Miodragović, R., Jovanović Todorović, M., Popović Djordjević, J. (2019): Mineral profile of the winter wheat grain: effects of soil tillage systems and nitrogen fertilization. - Applied Ecology and Environmental Research 17(5): 11757-11771. DOI: http://dx.doi.org/10.15666/aeer/1705_1175711771.

[16] Đokić, D. (1988): The role of nitrogen in the increase of wheat yield and quality improvement. - Agrohemija, Belgrade 5-6: $321-332$ (in Serbian).

[17] Egner, H., Riehm, H. (1955): Die Untersuchung von Böden. - In: Thun, R., Herrmann, R., Knickmann, E. (eds.) Methodenbuch Band I. Neumann Verlag, Radebeul/Berlin, pp. 110-125.

[18] Fagnano, M., Fiorentino, N., D’Egidio, M. G., Quaranta, F., Ritieni, A., Ferracane, R., Giampaolo Raimondi, G. (2012): Durum wheat in conventional and organic farming: yield amount and pasta quality in Southern Italy. - The Scientific World Journal 973058. DOI: 10.1100/2012/973058.

[19] Galieni, A., Stagnari, F., Visioli, G., Marmiroli, N., Speca, S., Angelozzi, G., D’Egidio, S., Pisante, M. (2016): Nitrogen fertilisation of durum wheat: a case study in Mediterranean area during transition to conservation agriculture. - Italian Journal of Agronomy 11: 12-23. DOI: 10.4081/ija.2016.662. 


$$
-185 \text { - }
$$

[20] García del Moral, L. F., Rharrabti, Y., Villegas, D., Royo, C. (2003): Evaluation of grain yield and its components in durum wheat under Mediterranean conditions: an ontogenic approach. - Agronomy Journal 95: 266-274.

[21] Gopinath, K. A., Saha, S., Mina, B. L., Pande, H., Kundu, S., Gupta, H. S. (2008): Influence of organic amendments on growth, yield and quality of wheat and on soil properties during transition to organic production. - Nutr. Cycling in Agroecosystems 82: 51-60. DOI 10.1007/s10705-008-9168-0.

[22] Hirzel, J., Matus, I., Madariaga, R. (2010): Effect of split nitrogen applications on durum wheat cultivars in volcanic soil. - Chilean Journal of Agricultural Research 70(4): 590595.

[23] ISO 10693 (1995): Soil Quality-Determination of Carbonate Content-Volumetric Method. - International Organization for Standardization, Genève.

[24] ISO 14235 (1998): Soil Quality-Determination of Organic Carbon by Sulfochromic Oxidation. - International Organization for Standardization, Genève.

[25] ISO 11464 (2006): Soil Quality-Pretreatment of Samples for Physico-chemical Analysis. - International Organization for Standardization, Genève.

[26] ISO 10390 (2010): Soil Quality—Determination of pH. - International Organization for Standardization, Genève.

[27] ISO/IEC 17025 (2017): General Requirements for the Competence of Testing and Calibration Laboratories. - International Organization for Standardization, Genève.

[28] Kappen, H. (1929): Bodenazidität. - Springer Verlag, Berlin.

[29] Khan, P., Memon, M. Y., Imtiaz, M., Aslam, M. (2009): Response of wheat to foliar and soil application of urea at different growth stages. - Pakistan Journal of Botany 41(3): 1197-1204.

[30] Khan, A. A., Alam, M. A., Alam, M. K., Alam, M. J., Sarker, Z. I. (2013): Correlation and path analysis of durum wheat (Triticum turgidum L. var. Durum). - Bangladesh Journal of Agricultural Research 38(3): 515-521.

[31] Knežević, D., Paunović, A., Kondić, D., Radosavac, A., Laze, A., Kovačević, V., Mićanović, D. (2019): Variability in seed germination of barley cultivars (Hordeum vulgare L.) grown under different nitrogen application rates. - Acta Agriculturae Serbica 24(47): 61-69. 1. DOI: 10.5937/AASer1947061K.

[32] Kostić, M., Đokić, D. (1975): Influence of nitrogen given in the flowering stage of wheat on vegetation length and grain yield and quality. - Journal for Scientific Agricultural Research, Belgrade 101: 153-159 (in Serbian).

[33] Lammerts van Bueren, E., Struik, P., Jacobsen, E. (2002): Ecological aspects in organic farming and its consequences for an organic crop ideotype. - Netherlands Journal of Agricultural Science 50: 1-26.

[34] Langer, R. H. M. (1979): The dynamics of wheat yield. - New Zealand Wheat Review 14: 32-40.

[35] Li, Y. F., Wu, Y., Hernandez-Espinosa, N., Pena, R. J. (2013): Heat and drought stress on durum wheat: responses of genotypes, yield, and quality parameters. - Journal of Cereal Science 57: 398-404.

[36] López-Bellido, L., López-Bellido, R. J., Redondo, R. (2005): Nitrogen efficiency in wheat under rainfed Mediterranean conditions as affected by split nitrogen application. Field Crops Research 94: 86-97.

[37] Luković, K., Prodanović, S., Perišić, V., Milovanović, M., Perišić, V., Rajičić, V., Zečević, V. (2020): Multivariate analysis of morphological traits and the most important productive traits of wheat in extreme wet conditions. - Applied Ecology and Environmental Research 18(4): 5857-5871. DOI: http://dx.doi.org/10.15666/aeer/1804_58575871,

[38] Lupini, A., Preiti, G., Badagliacca, G., Abenavoli, M. R., Sunseri, F., Monti, M., Bacchi, M. (2021): Nitrogen use efficiency in durum wheat under different nitrogen and water 


$$
-186-
$$

regimes in the Mediterranean Basin. - Frontiers in Plant Science 11: 607226. DOI: 10.3389/fpls.2020.607226.

[39] Mariem, S. B., González-Torralba, J., Collar, C., Aranjuelo, I., Morales, Fermín (2020): Durum wheat grain yield and quality under low and high nitrogen conditions: insights into natural variation in low- and high-yielding genotypes. - Plants (Basel) 9(2) 1636. DOI: 10.3390/plants9121636.

[40] Mascianica, M. P., Valden, R. F. (1986): Performans of winter wheat under conventional and intensive N management. - Journal of Applied Agricultural Research 1: 32-36.

[41] Matković, M., Bošković, J., Zečević, V., Knežević, D., Đurić, N. (2015): Influence of genotype on yield and quality components of durum wheat in organic production. Proceedings. Fifth International Symposium on Natural Resources Management. May $23^{\text {rd }}$, Faculty of Management, Zaječar, Republic of Serbia, pp. 17-22.

[42] Matković Stojšin, M., Zečevć, V., Petrović, S., Dimitrijević, M., Mićanović, D., Banjac, B., Knežević, D. (2018): Variability, correlation, path analysis and stepwise regression for yield components of different wheat genotypes. - Genetika 50(3): 817-828.

[43] Michigan State University (1990): User's Guide to MSTAT-C. - Michigan State University, Michigan.

[44] Mohammadi, M., Karimizadeh, R., Mohammad Kazem Shefazadeh, M. K., Sadeghzadeh, B. (2011): Statistical analysis of durum wheat yield under semi-warm dryland condition. - Australian Journal of Crop Science 5(10): 1292-1297.

[45] Nofouzi, F. (2018): Evaluation of seed yield of durum wheat (Triticum durum) under drought stress and determining correlation among some yield components using path coefficient analysis. - UNED Research Journal 10(1): 179-183. https://www.scielo.sa.cr/pdf/cinn/v10n1/1659-4266-cinn-10-01-179.pdf.

[46] Orcen, N., Tosun, M., Irget, E. (2013): Effect of nitrogen fertilizer timing and source on some yield and quality parameters of durum wheat (Triticum durum). - Journal of Food Agriculture and Environment 11: 943-948.

[47] Rao, B. N., Pozniak, C. J., Hucl, P. J., Briggs, C. (2010): Baking quality of emmerderived durum wheat breeding lines. - Journal of Cereal Science 51: 299-304.

[48] Sarto, M. V. M., Sarto, J. R. W., Rampim, L., Rosset, J. S., Bassegio, D., Costa, P. F., Inagaki, A. M. (2017): Wheat phenology and yield under drought: a review. - Australian Journal of Crop Science 11(08): 941-946. DOI: 10.21475/ajcs.17.11.08.pne351.

[49] Staugaitis, G., Aleknavičienè, L., Brazienė, Z., Marcinkevičius, A., Paltanavičius, V. (2017): The influence of foliar fertilization with nitrogen, sulphur, amino acids and microelements on spring wheat. - Zemdirbyste-Agriculture 104(2): 123-130. DOI: 10.13080/z-a.2017.104.016.

[50] Tedone, L., Verdini, L., Grassano, N., Tarraf, W., De Mastro, G. (2014): Optimising nitrogen in order to improve the efficiency, eco-physiology, yield and quality on one cultivar of durum wheat. - Italian Journal of Agronomy 9: 49-54. DOI: 10.4081/ija.2014.536.

[51] Tedone, L., Ali, S. A., De Mastro, G. (2017): Optimization of Nitrogen in Durum Wheat in the Mediterranean Climate: The Agronomical Aspect and Mediterranean Climate: The Agronomical Aspect and Greenhouse Gas (GHG) Emissions. - In: Amanullah, Fahad, S. (eds.) Nitrogen in Agriculture. IntechOpen, London, pp. 131-162. http://dx.doi.org/10.5772/intechopen.70195.

[52] Trnka, M., Reimund, P., Rötter, R. P., Ruiz-Ramos, M., Kersebaum, K. C., Olesen, J. E., Žalud, Z., Semenov, M. A. (2014): Adverse weather conditions for European wheat production will become more frequent with climate change. - Nature Climate Change 4: 637-643. https://doi.org/10.1038/nclimate2242.

[53] Van Reeuwijk, L. P. (ed) (2002): Procedures for Soil Analysis. Sixth Ed. - ISRIC FAO Technical Paper Vol. 9. International Soil Reference and Information Centre, Wageningen, pp 12-16. 


$$
-187 \text { - }
$$

[54] Woolfolk, C. W., Raun, W. R., Johnson, G. V., Thomason, W. E., Mullen, R. W., Wynn, K. J., Freeman, K. W. (2002): Influence of late-season foliar nitrogen applications on yield and grain nitrogen in winter wheat. - Agronomy Journal 94(3): 429-434. https://doi.org/10.2134/agronj2002.4290.

[55] Zadoks, J. C., Chang, T. T., Konzak, C. F. (1974): A decimal code for the growth stages of cereals. - Weed Research 14: 415-421.

[56] Zečević, V., Đokić, D., Knežević, D., Mićanović, D. (2004): The influence of nitrogen foliar applications on yield and bread making quality parameters of wheat. - Kragujevac Journal of Science 26: 89-90.

[57] Zhang, M., Wang, H., Yi, Y., Ding, J., Zhu, M., Li, C., Guo, W., Feng, C., Zhu, X. (2017): Effect of nitrogen levels and nitrogen ratios on lodging resistance and yield potential of winter wheat (Triticum aestivum L.). - PLoS ONE 12(11): e0187543. https://doi.org/10.1371/journal.pone.0187543. 